\title{
KURZER BERICHT ÜBER DIE PROBLEME DES HÖHLENTOURISMUS IN JUGOSLAWIEN
}

\author{
France Habe*
}

\begin{abstract}
SUMMARY [Short note on the problems of the Yugoslav show caves ]
\end{abstract}
In the frame of theIUS, the activities concerning the Yugoslav show caves are reported and the most relevant problems are emphasized.

\section{RIASSUNTO [Breve nota sui problemi delle grotte turistiche in Jugoslavia]}

Nell'ambito dell'UIS, vengono descritte le attività riguardanti le grotte turistiche jugoslave evidenziando $\mathrm{i}$ problemi più rilevanti.

Als Vorsitzender des Departements für den Karstschutz und Schauhöhlen bei der Union Internationale de Spéléologie und zugleich in Jugoslawien will ich kurz berichten, was sich in dieser Hinsicht in Jugoslawien und besonders in der Republik Slowenien verändert hat. Wie bekannt, ist die Skocjanske jame bei Divaca die einzige europäische Schauhöhle, die in die Liste die Natur-und Kulturerbes bei der UNESCO im Jahre 1986 eingetragen wurde. Leider werden diese Höhlen durch die noch immer starke Verschmutzung des Ponorflusses Reka (Er fließt vor der Höhle in der III. oder sogar in der IV Qualitätsklasse).

Die Kommission für den Höhlenschutz und Schauhöhlen bei der speläologischen Föderation Jugoslawiens veranstaltete deswegen schon das zweite Symposium im Mai 1990 in Sezana, wo neben den allgemeinen Problemen des Karstschutzes hauptsächlich zwei Probleme angeschnitten wurden : die Sanierung des Ponorflusses Reka (Timavo), die nach dem OsimoVertrag mit Italien ein internationales Problem bedeutet.

Nach dem Beschluss von UNESCO muss die Sanierung des Flusses Reka bis zum Ende des Jahres 1991 vollendet werden.

Anbei eine Bemerkung: leider wird auch der berühmte Grottenmolc Proteus anguinus in der Höhle von Postojna wegen der Verunreinigung des Ponorflusses Pivka bedroht.

In Jugoslawien gibt es augenblicklich 42 Schauhöhlen, von denen sind 16 in der Republik Slowenien, unter denen zwei Weltschauhöhlen, die Höhle von Postojna und Skocjanske jame. Leider werden alle diese Höhlen verschieden betreut. So werden diese Schauhöhlen stand den verschieden wirtschaftlichen und anderen nicht fachmännischen Organisationen geführt. Nur 6 Schauhöhlen sind in der Hand der Höhlenforschervereine. Gerade die Frage der Verwaltung der Schauhöhlen war am II. jugosl. Symposium in Sezana im Vordergrund.

Darum wurde dabei beschlossen, dass es dringend nötig sei im Rahmen der einzelnen Republiken einen einheitlichen Fachkörper zu gründen,

\footnotetext{
* Vojkova 2, Postbox 44, SLO 66230 Postojna (Slovenia)
} 
der die Aufgabe hätte, eine einheitliche Richtung zum Zwecke der Eröffnung, der Infrastruktur, Führung und Propaganda der Höhlen zu organisieren. Dass es dringend nötig ist, eine einheitliche Organisation zu gründen, beweisst der unglückliche Tod einer jungen Biologin in der Planina-Höhle am Rande des Planinsko polje am 27 Oktober 1991, aufgrund der nicht ausreichenden Vorschriften über den Besuch der Höhle. Diese vorgeschlagene republikanische Fachkörper sollen von den fachmännischen speläologischen, touristischen und Umweltschutz $=$ organisationen gebildet werden.

In der letzten Zeit plant die neue slowenische Demokratie bei den beiden slowenischen Weltschauhöhlen- bei der Höhle von Postojna und Skocjanske jame, ein Consortium, einen Höhlenrat oder eine Höhlen= kommission zu gründen, die schon 1824 die leitende Rolle bei der Höhle von Postojna spielte.

Schon heute verzeichnet die Postojnska jama jährlich bis 900.000 Besucher. Diese Zahl wird sich bestimmt noch erhöhen können, wenn es uns gelingen wird, die Industrie wie auch die lästigen Kasernen, die stark zur Verunreinigung des klassischen Karstes beitragen, schnell abzuschaffen. 